\title{
14. CAMPANIAN TO MAESTRICHTIAN DINOFLAGELLATE CYSTS FROM THE UNITED STATES ATLANTIC MARGIN, DEEP SEA DRILLING PROJECT SITE $612^{1}$
}

\author{
Bruce A. Tocher, Department of Geological Sciences, Plymouth Polytechnic, Plymouth ${ }^{2}$
}

\begin{abstract}
Fifty-nine samples from the basal $110 \mathrm{~m}$ of DSDP Hole 612 (United States Atlantic Margin) were analyzed for palynomorph content. In total, 84 species and subspecies of dinoflagellate cysts were recorded which, on comparison with published data and shipboard analyses, indicate a Campanian to Maestrichtian age for this part of the succession. The Campanian/Maestrichtian contact is taken to occur in the upper part of Core 612-69.
\end{abstract}

\section{INTRODUCTION}

Leg 95 of the Deep Sea Drilling Project (DSDP) drilled at two sites, 612 and 613 , on the New Jersey middle slope and upper rise, respectively. Site 612 was selected to provide a stratigraphic section which would serve as a link between the COST B-3 Well on the upper slope and DSDP Site 605 on the upper rise (Fig. 1). One of the primary aims was to provide a complete Upper Cretaceous and Cenozoic section for biostratigraphic analysis of this part of the margin. This chapter deals only with Upper Cretaceous material. A full report on the Cenozoic part of the section will follow at a later date.

There have been several previous studies of Campanian to Maestrichtian dinoflagellate cysts. In Europe, significant work was carried out by Deflandre $(1935,1936$, 1937), Lejeune-Carpentier (1938, 1939), Alberti (1959, 1961), and, more recently, by Clarke and Verdier (1967), Kjellstrom (1973), Wilson (1971, 1974), and Hansen (1977). In Australia, there was a series of publications by Cookson and Eisenack (1958, 1960, 1962, 1968, 1970, 1974), and in New Zealand Wilson published a series (1976a, 1976b, 1983, 1984). Notable studies from the United States and Canada include those of Drugg (1967), Harland (1973), Williams (1975), Williams and Brideaux (1975), McIntyre (1975), Benson (1976), Bujak and Williams (1978), May (1980), and Whitney (1984).

In total, 59 samples were processed for this study using standard palynological techniques (Neves and Dale, 1963; Doher, 1980). Eighty-four species and subspecies of dinoflagellate cysts were recorded (see Appendix); their distribution is plotted in Figure 2.

\section{BIOSTRATIGRAPHY}

\section{Site 612}

The basal 25 to $26 \mathrm{~m}$ of the section (below Sample 612-69-2, 43-45 cm; Fig. 2) contains 48 species and subspecies of cysts, 19 of which are restricted to this inter-

\footnotetext{
${ }^{1}$ Poag, C. W., Watts, A. B., et al., Init. Repts. DSDP, 95: Washington (U.S. Govt. Printing Office).

2 Address: Department of Geological Sciences, Plymouth Polytechnic, Drake Circus, Plymouth, Devon PL4 8AA, U.K.
}

val. These include the last occurrences of Palaeohystrichophora infusorioides Deflandre, Odontochitina costata Alberti, Trichodinium castaneum (Deflandre) Clarke and Verdier, and Odontochitina operculata (O. Wetzel) Deflandre and Cookson. Also, Dinogymnium microgranulosum Clarke and Verdier, D. digitus (Deflandre) Evitt et al., D. cf. euclaense Cookson and Eisenack, Kleithriasphaeridium truncatum (Benson) Stover and Evitt, Cannosphaeropsis utinensis $\mathrm{O}$. Wetzel, and Odontochitina porifera Cookson have their first occurrences in this part of the section. A number of those occurrences compare closely with the zonation proposed by Bujak and Williams (1978) on the basis of studies of offshore eastern Canada. In particular, the last occurrences of $P$. infusorioides, $O$. costata, $O$. operculata, and $T$. castaneum and the first occurrence of $D$. digitus are indicative of Bujak and Williams's (1978) O. operculata Assemblage Zone of Campanian age. Furthermore, Williams (1975) and Wilson (1984) suggest that $C$. utinensis and $O$. porifera, respectively, first occur in the Campanian. The record most similar to the cyst distribution at Site 612, however, is that from the Atlantic Highlands, New Jersey (May, 1980). May (1980) suggests that the last occurrences of $P$. infusorioides, $O$. costata, and Xenascus ceratioides (Deflandre) Lentin and Williams, coinciding with the first occurrences of Samlandia? angustivela (Deflandre and Cookson) Eisenack and C. utinensis, indicate a latest Campanian to earliest Maestrichtian age. The close agreement of Site 612 results with these records therefore strongly suggests that the sediments below Sample 612-69-2, 43-45 cm (Fig. 2) are of late Campanian age.

The remaining $80 \mathrm{~m}$ of section examined here (from Sample 612-69-2, 43-45 cm to Sample 612-61-1, 40-42 cm; Fig. 2) contain 60 species and subspecies of cysts, 36 of these occurring for the first time. Stratigraphically significant occurrences include the last appearances of $C y$ clonephelium distinctum Deflandre and Cookson and $X$. ceratioides near the base of this interval, and the incoming of S.? angustivela, Ceratiopsis diebelii (Alberti) Vozzhennikova, Cordosphaeridium fibrospinosum Davey and Williams, $C$. varians May, Spongodinium delitiense (Ehrenberg) Deflandre, and Dinogymnium westralium (Cookson and Eisenack) Evitt et al., and, high- 


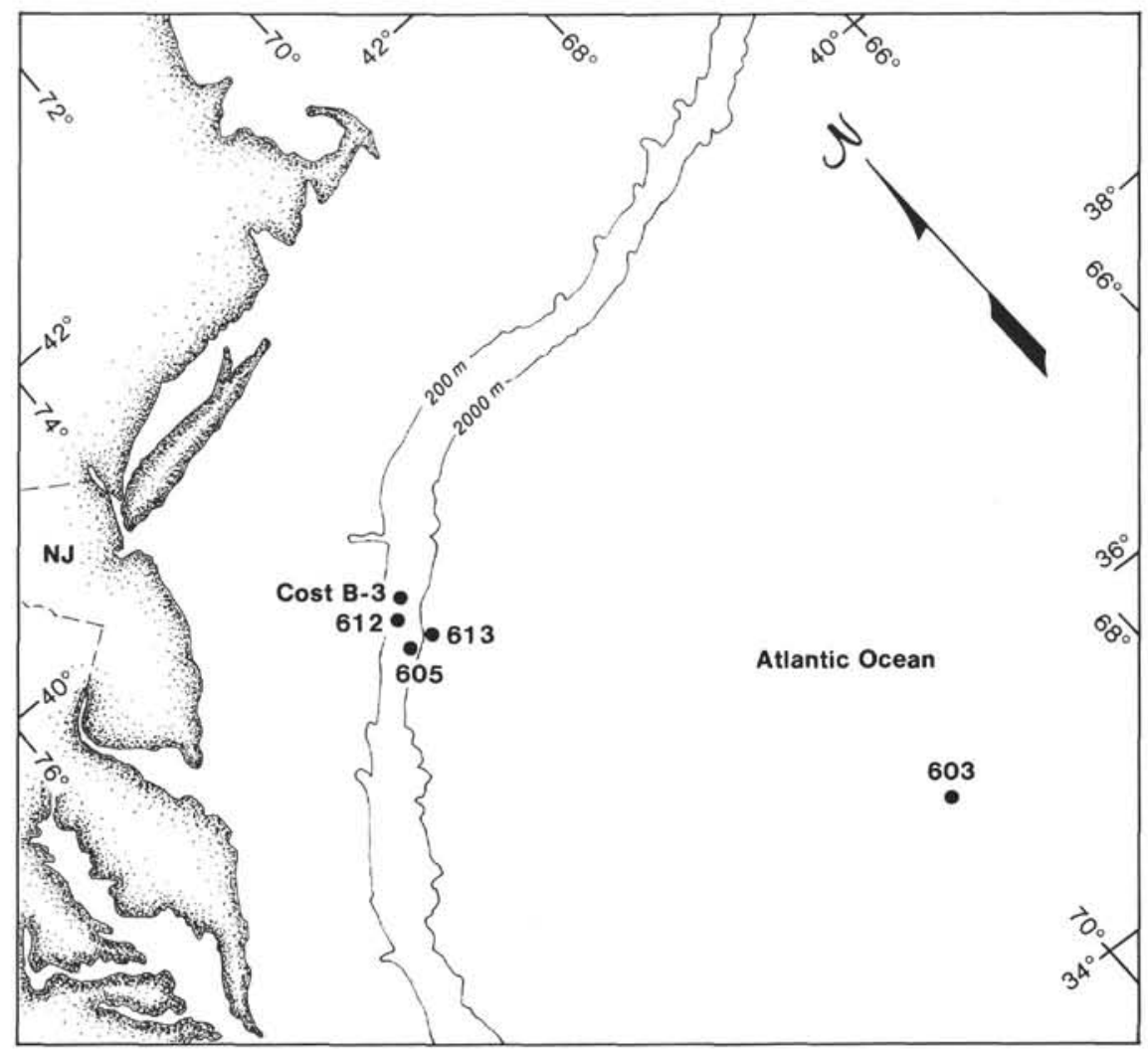

Figure 1. Location of Site 612, offshore New Jersey (NJ), on the western Atlantic margin, U.S.A.

er in the section, Phelodinium tricuspis (O. Wetzel) Stover and Evitt, Spiniferites cornutus (Gerlach) Sarjeant, and Palaeocystodinium australinum (Cookson) Lentin and Williams. Several of these forms, in particular C. $f i$ brospinosum, $C$. diebelii, $P$. tricuspis, $P$. australinum, and $S$. delitiense, are recorded by Bujak and Williams (1978) as being characteristic of their $D$. euclaense Assemblage Zone of Maestrichtian age (although May [1980] records $C$. fibrospinosum as first appearing the late Campanian). Both Wilson (1974) and May (1980) record the incoming of S.? angustivela and $S$. delitiense as occurring in the lower Maestrichtian, and the latter author also regards the first occurrences of $C$. diebelii and $D$. westralium as indicating a similar age. It has been suggested (Wilson, 1984; Hansen, 1977) that $P$. australinum and $S$. cornutus are upper Maestrichtian indicators. However, May (1980) records the former species from the lower Maestrichtian of New Jersey, and it is suggested that the range of $S$. cornutus can likewise be extended down to this level. The absence of any definite upper Maestrichtian species suggests that the sediments are most reliably assigned to the lower Maestrichtian, and this is in agreement with the shipboard analyses based on foraminifers and nannofossils.

The Campanian/Maestrichtian contact is taken to occur in the upper part of Core 612-69, and it is associated with a sharp lithologic break from dark gray foraminiferal shale (below) to marly foraminiferal nannofossil chalk.

\section{SUMMARY}

In total, 84 species and subspecies of dinoflagellate cysts are recorded from the basal $110 \mathrm{~m}$ of DSDP Hole 612. On comparison with published dinoflagellate cyst records, these are taken to indicated a late Campanian to early Maestrichtian age for this part of the section. This conclusion is further strengthened by shipboard analyses based on foraminifers and nannofossils (site chapters, this volume). The Campanian/Maestrichtian contact is placed in the upper part of Core 612-69 and is associated with a sharp lithologic break.

\section{ACKNOWLEDGMENTS}

I would like to thank Mr. R. Emmett for processing the samples, and Plymouth Polytechnic Media Services Unit for producing the photographs. This work was completed under the tenure of an LEA Research Fellowship at the Dept. of Geological Sciences, Plymouth Polytechnic, receipt of which is gratefully acknowledged.

\section{REFERENCES}

Alberti, G., 1959. Zur Kenntnis der Gattung Deflandrea Eisenack (Dinoflag.) in der Kreide und in Alttertiar Nord- und Mitteldeutschlands. Mitt. Geol. Staatsinst. Hamburg, 28:93-105.

, 1961. Zur Kenntnis mesozoischer und Alterttiarer Dinoflagellaten und Hystrichosphaerideen von Nord- und Mitteldeutschland sowie einigen anderen europaischen Gebieten. Palaeontographica, Abt. A, 116:1-58.

Benson, G. D., 1976. Dinoflagellate biostratigraphy of the CretaceousTertiary boundary, Round Bay, Maryland. Tulane Stud. Geol. Paleontol., 12(4):169-228. 
Bujak, J. P., and Williams, G. L., 1978. Cretaceous palynostratigraphy of offshore southeastern Canada. Bull. Geol. Surv. Can., 297.

Clarke, R. F. A., and Verdier, J.-P., 1967. An investigation of microplankton assemblages from the chalk of the Isle of Wight, England. Verh. K. Ned. Akad. Wet., Afd. Nat. Eerste Reeks, 24: $1-96$.

Cookson, I. C., and Eisenack, A., 1958. Microplankton from Australian and New Guinea upper Mesozoic sediments. Proc. R. Soc. Victoria, 70:19-79.

1960. Microplankton from Australian Cretaceous sediments. Micropalaeontology, 6:1-18.

1962. Additional microplankton from Australian Cretaceous sediments. Micropalaeontology, 8:485-507.

1968. Microplankton from two samples from Gingin Brook No. 4 Borehole, Western Australia. J. R. Soc. W. Austral., 51: $110-122$.

, 1970. Cretaceous microplankton from the Eucla Basin, Western Australia. Proc. R. Soc. Victoria, 83:137-157.

, 1974. Microplankton aus Australischen Mesozoischen und Tertiaren Sedimenten. Palaeontographica, Abt. B, 148:44-93.

Doher, L. I., 1980. Palynomorph preparation procedures currently used in the palaeontology and stratigraphy laboratories, U.S. Geological Survey. U.S. Geol. Surv. Circ., 830.

Deflandre, G. 1935. Considérations biologique sur les microorganismes d'origine planctonique conservés dans les silex de la craie. Bull. Biol. Fr. Belg., 69:213-244.

, 1936. Microfossils des silex crétacés. Premier partie. Générilités Flagelles. Ann. Paleontol., 25:151-191.

, 1937. Microfossiles des silex crétacés. Deuxième partie. Flagelles incertae sedis Hystrichosphaerides. Sarcodines organisms divers. Ann. Paleontol., 26:51-103.

Drugg, W. S., 1967. Palynology of the upper Moreno Formation (Late Cretaceous-Palaeocene), Escarpado Canyon, California. Palaeontographica, Abt. B, 120:1-71.

Hansen, J. M., 1977. Dinoflagellate stratigraphy and echinoid distribution in upper Maestrichtian and Danian deposits from Denmark. Bull. Geol. Soc. Denmark, 26:1-26.

Harland, R., 1973. Dinoflagellate cysts and acritarchs from the Bearpaw Formation (upper Campanian) of southern Alberta, Canada. Palaeontology, 16:665-706.

Kjellstrom, G., 1973. Maestrichtian microplankton from the Hollviken Borehole No. 1 in Scania, southern Sweden. Sver. Geol. Unders., Afh. Och Uppsatser, 67:1-59.

Lejeune-Carpentier, M., 1938. L'étude microscopique des silex. Areoligera: nouveau genre d'Hystrichosphaeridee (Sixième note). Ann. Soc. Geol. Belg., 62:163-174.

1939. Létude microscopique des silex. Un nouveau Peridinien créticique: Gonyaulax wetzeli (Septième note). Ann. Soc. Geol. Belg., 62:525-529.

McIntyre, D. J., 1975. Morphologic changes in Deflandrea from a Campanian section, District of Mackenzie, N. W. T., Canada. Geosci. Man., 11:61-76.

May, F. E., 1980. Dinoflagellate cysts of the Gymnodiniaceae, Peridiniaceae, and Gonyaulacaceae from the Upper Cretaceous Monmouth Group, Atlantic Highlands, New Jersey. Palaeontographica, Abt. B, 172:10-116.

Neves, R., and Dale, B., 1963. A modified filtration system for palynological preparations. Nature, 198:775-776.

Whitney, B. L., 1984. Dinoflagellate biostratigraphy of the Maestrichtian-Danian section in southern Maryland. In Frederikson, N. O., and Krafft, K. (Eds.), A.A.S.P. Field Trip Volume and Guidebook to the Cretaceous and Tertiary Stratigraphy, Palaeontology, and Structure, Southeastern Maryland and Northeastern Virginia: Reston, VA (U.S.G.S.), pp. 123-136.

Williams, G. L., 1975. Dinoflagellate and spore stratigraphy of the Mesozoic-Cenozoic, offshore eastern Canada. Pap. Geol. Surv. Can., 74-30 (2):107-161.

Williams, G. L., and Brideaux, W. W., 1975. Palynologic analyses of upper Mesozoic and Cenozoic rocks of the Grand Banks, Atlantic continental margin. Bull. Geol. Surv. Can., 236:1-163.

Wilson, G. J., 1971. Observations on European Late Cretaceous dinoflagellate cysts. Proc. Second Planktonic Conference (Roma), pp. $1259-1275$.
1974. Upper Campanian and Maestrichtian dinoflagellate cysts from the Maastricht region and Denmark [Ph.D. dissert.]. Nottingham Univ.

1976a. Late Cretaceous (Senonian) dinoflagellate cysts from the Kahuitasa Tuff, Chatham Islands. N. Z. J. Geol. Geophys., 19: 127-130.

1976b. An Albian-Cenomanian dinoflagellate assemblage from the Mokoiwi Siltstone, Mt. Taitai, Raukumara Range, New Zealand. N. Z. J. Geol. Geophys., 19:132-136.

1984. New Zealand Late Jurassic to Eocene dinoflagellate biostratigraphy—a summary. Newsl. Stratigr., 13(2):104-117.

Date of Initial Receipt: 10 June 1985

Date of Acceptance: 8 October 1985

\section{APPENDIX \\ List of Taxa}

Achomosphaera crassipellis (Deflandre and Cookson, 1955) Stover and Evitt, 1978

Achomosphaera ramulifera (Deflandre, 1937) Evitt, 1963

Achomosphaera sagena Davey and Williams, 1966

Alterbia acutula (Wilson, 1967) Lentin and Williams, 1976

Andalusiella spicata (May, 1980) Lentin and Williams, 1981

Areoligera senonensis Lejeune-Carpentier, 1938

Bacchidinium sp.?

Cannosphaeropsis utinensis O. Wetzel, 1933; emend. Duxbury, 1980

Ceratiopsis diebelii (Alberti, 1959) Vozzhennikova, 1967

Ceratiopsis sp. A

Ceratiopsis sp. B

Chlamydophorell discreta Clarke and Verdier, 1967

Cordosphaeridium fibrospinosum Davey and Williams, 1966

Cordosphaeridium varians May, 1980

Coronifera oceanica Cookson and Eisenack, 1958

Coronifera striolata (Deflandre, 1937) Stover and Evitt, 1978

Cribroperidinium edwardii (Cookson and Eisenack, 1958) Stover and Evitt, 1978

Cribroperidinium sp.

Cyclonephelium distinctum Deflandre and Cookson, 1955

Cyclonephelium expansum Corradini, 1973

Dapsilidinium? pumilum (Davey and Williams, 1966) Lentin and Williams, 1981

Dinogymnium digitus (Deflandre, 1935) Evitt et al., 1967

Dinogymnium cf. euclaense Cookson and Eisenack, 1970

Dinogymnium lanceolatum May, 1977

Dinogymnium microgranulosum Clarke and Verdier, 1967

Dinogymnium westralium (Cookson and Eisenack, 1958) Evitt et al., 1967; emend. May, 1977

Dinogymnium sp. A

Dinogymnium sp. B

Dinopterygium sp.?

Eurydinium ingramii (Cookson and Eisenack, 1970) Stover and Evitt, 1978

Eurydinium sp.

Exochosphaeridium bifidum (Clarke and Verdier, 1967) Clarke et al., 1968

Glaphrocysta ordinata (Williams and Downie, 1966) Stover and Evitt, 1978

G. retiintexta (Cookson, 1965) Stover and Evitt, 1978

Gonyaulacysta? wetzelii (Lejeune-Carpentier, 1939) Sarjeant, 1969

Hystrichokolpoma unispinum Williams and Downie, 1966a

Hystrichokolpoma sp.

Hystrichodinium pulchrum pulchrum Deflandre, 1935

Hystrichosphaeridium palmatum (White, 1842) Downie and Sarjeant, 1965

Hystrichosphaeridium tubiferum (Ehrenberg, 1838) brevispinum (Davey and Williams, 1966) Lentin and Williams, 1973

Hystrichosphaeridium tubiferum tubiferum (Ehrenberg, 1838) Deflandre, 1937; emend. Davey and Williams, 1966

Hystrichosphaeridium sp.

Hystrichostrogylon membraniphorum Agelopoulos, 1964 


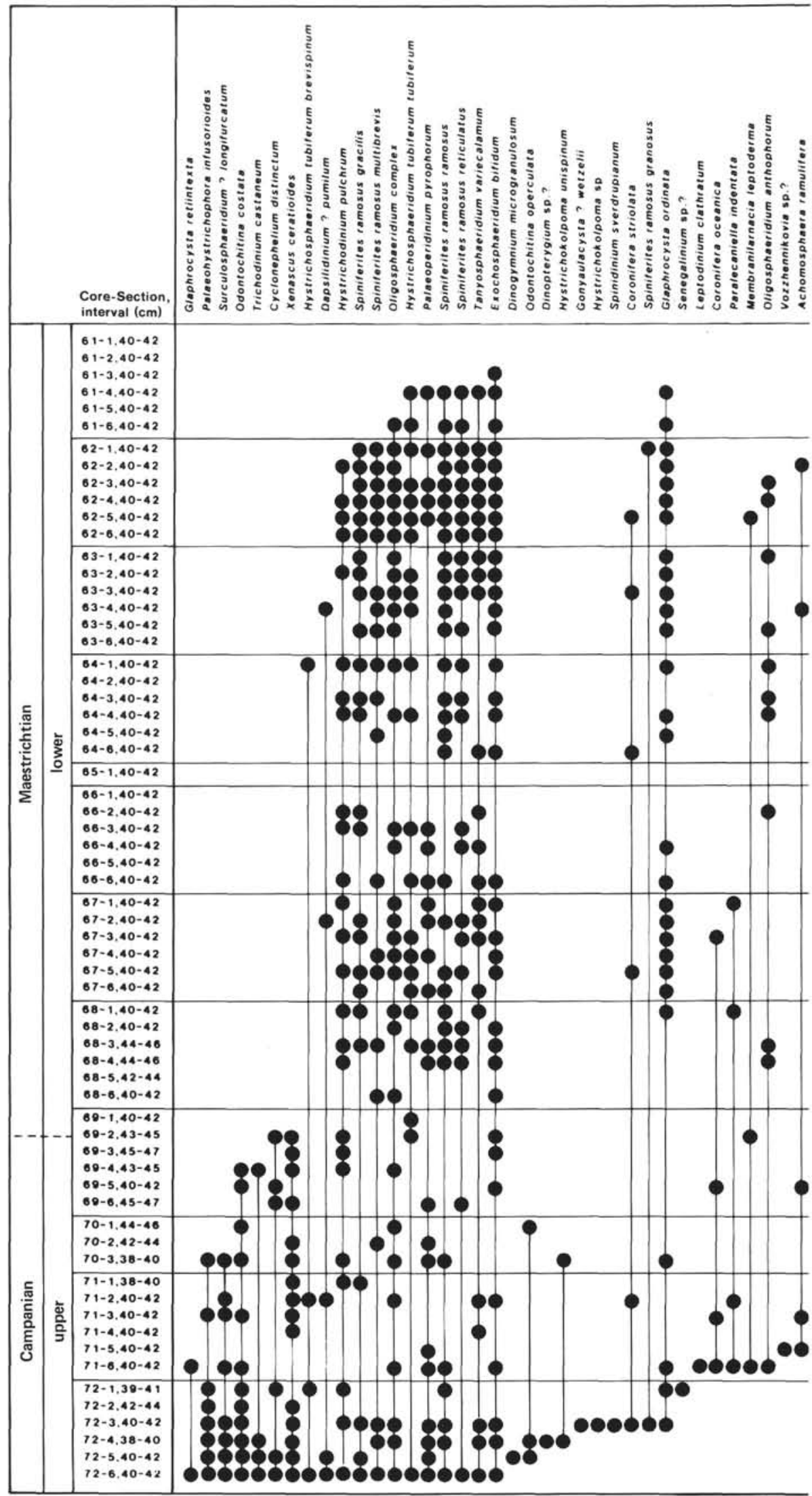

Figure 2. Range and distribution chart of dinoflagellate cysts recovered from Campanian to Maestrichtian sediments of Hole 612 . 


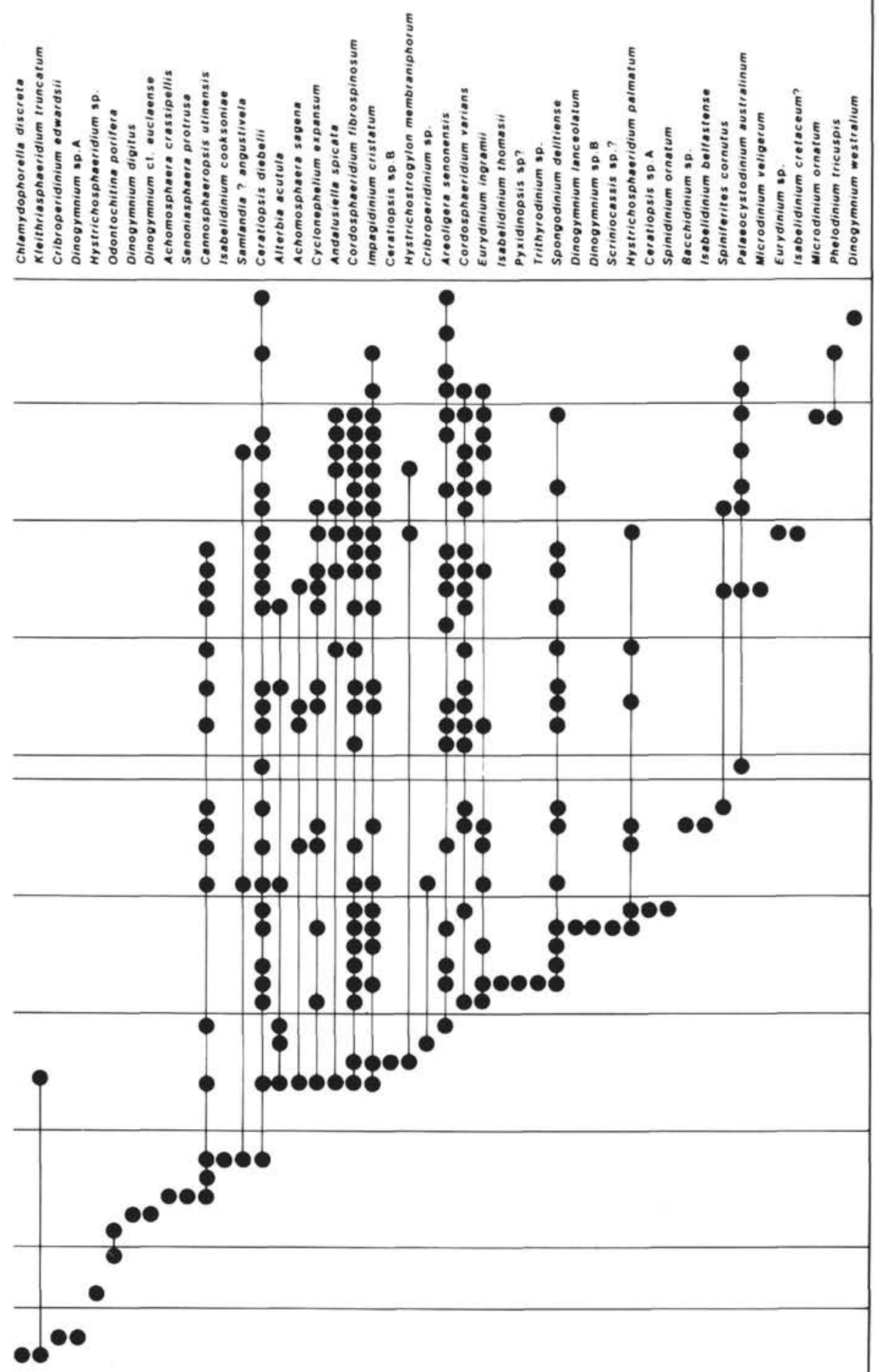

Figure 2 (continued). 


\section{B. A. TOCHER}

Impagidinium cristatum (May, 1980) Lentin and Williams, 1981

Isabelidinium belfastense (Cookson and Eisenack, 1961) Lentin and Williams, 1977

Isabelidinium cooksoniae (Alberti, 1959) Lentin and Williams, 1977 Isabelidinium cretaceum? (Cookson, 1956) Lentin and Williams, 1977

Isabelidinium thomasii (Cookson and Eisenack, 1961) Lentin and Williams, 1977

Kleithriasphaeridium truncatum (Benson, 1976) Stover and Evitt, 1978

Leptodinium clathratum (Cookson and Eisenack, 1960) Sarjeant, 1969 Membranilarnacia leptoderma (Cookson and Eisenack, 1958) Eisenack, 1963

Microdinium ornatum Cookson and Eisenack, 1960

Microdinium veligerum (Deflandre, 1937) Davey, 1969

Odontochitina costata Alberti, 1961; emend. Clarke and Verdier, 1967

Odontochitina operculata (O. Wetzel, 1933) Deflandre and Cookson, 1955

Odontochitina porifera Cookson, 1956

Oligosphaeridium anthophorum (Cookson and Eisenack, 1958) Davey, 1969

Oligosphaeridium complex (White, 1842) Davey and Williams, 1966

Palaeocystodinium australinum (Cookson, 1965) Lentin and Williams, 1976

Palaeohystrichophora infusorioides Deflandre, 1935

Palaeoperidinium pyrophorum (Ehrenberg, 1838) Sarjeant, 1967

Paralecaniella indentata (Deflandre and Cookson, 1955) Cookson and Eisenack, 1970; emend. Elsik, 1977
Phelodinium tricuspis (O. Wetzel, 1933) Stover and Evitt, 1978

Pyxidinopsis $\mathrm{sp}$.?

Samlandia? angustivela (Deflandre and Cookson, 1955) Eisenack, 1963

Scriniocassis $\mathrm{sp} . ?$

Senegalinium sp.?

Senoniasphaera protrusa Clarke and Verdier, 1967

Spinidinium ornatum (May, 1980) Lentin and Williams, 1981

Spindinium sverdrupianum (Manum, 1963) Lentin and Williams, 1973

Spiniferites cornutus (Gerlach, 1961) Sarjeant, 1970

Spiniferites ramosus (Ehrenberg, 1838) gracilis (Davey and Williams, 1966) Lentin and Williams, 1973

Spiniferites ramosus (Ehrenberg, 1838) granosus (Davey and Williams, 1966) Lentin and Williams, 1973

Spiniferites ramosus (Ehrenberg, 1838) multibrevis (Davey and Williams, 1966) Lentin and Williams, 1973

Spiniferites ramosus ramosus (Ehrenberg, 1838) Loeblich and Loeblich, 1966

Spiniferites ramosus (Ehrenberg, 1838) reticulatus (Davey and Williams, 1966) Lentin and Williams, 1973

Spongodinium delitiense (Ehrenberg, 1838) Deflandre, 1936

Surculosphaeridium? longifurcatum (Firtion, 1952) Davey et al., 1966

Tanyosphaeridium variecalamum Davey and Williams, 1966

Trichodinium castaneum (Deflandre, 1935) Clarke and Verdier, 1967

Trithyrodinium $\mathrm{sp}$.

Vozzhennikovia sp.?

Xenascus ceratioides (Deflandre, 1937) Lentin and Williams, 1973 


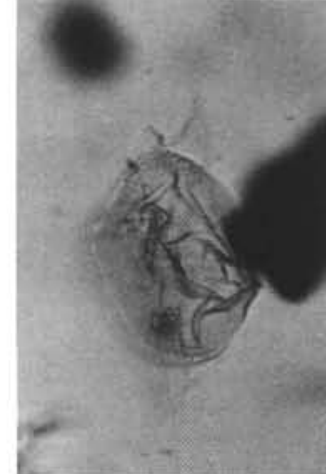

1

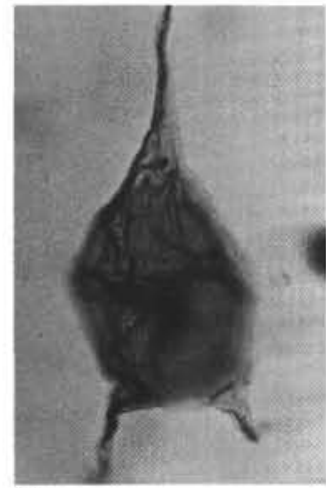

2

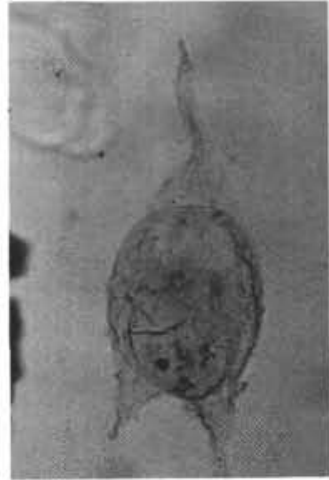

3

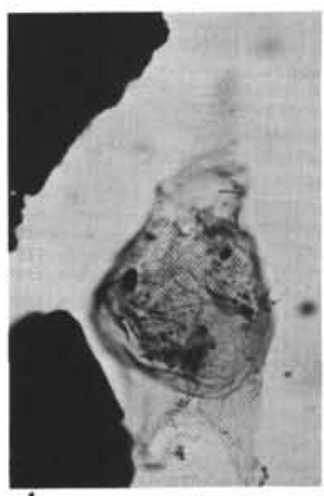

4

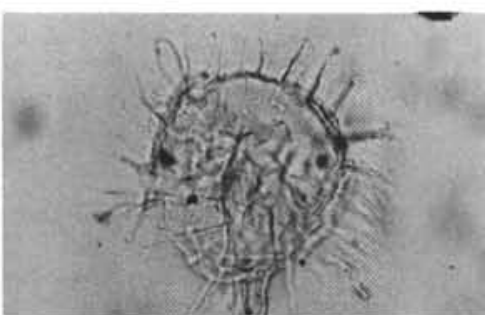

5

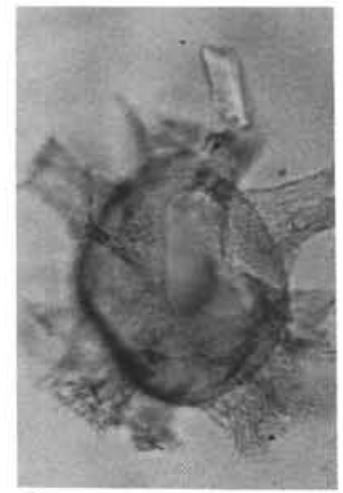

8

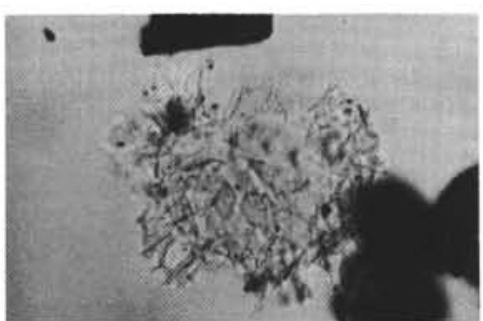

6

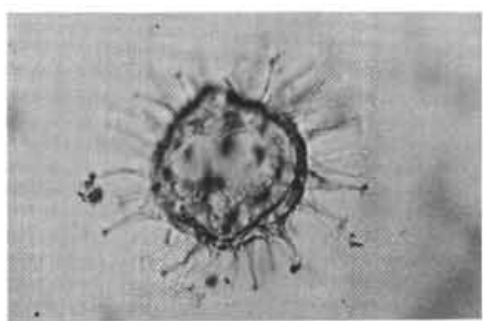

7

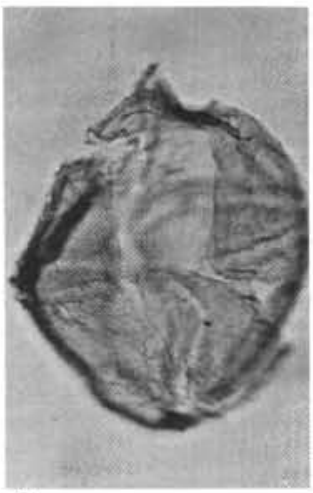

9

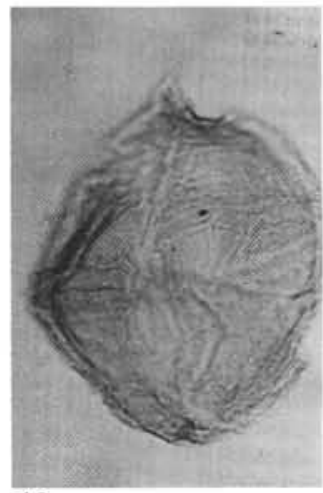

10

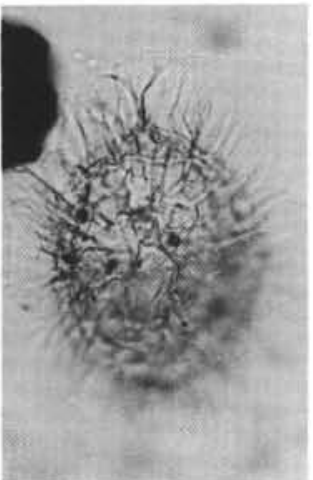

11

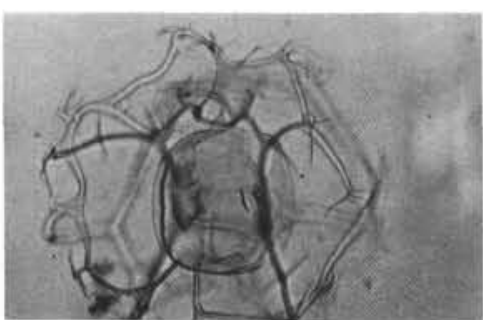

12

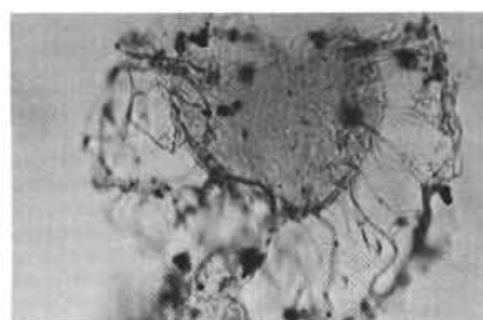

13

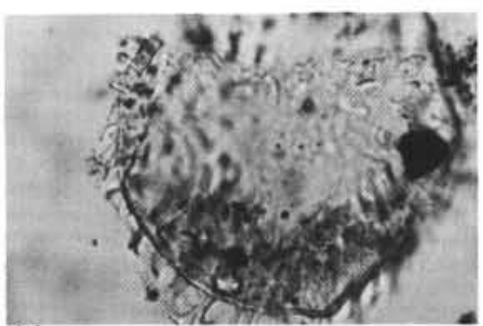

14

Plate 1. (Specimens photographed at $\times 400$ unless otherwise stated. All specimens photographed under normal transmitted light.) 1. Andalusiella spicata (May) Lentin and Williams, Sample 612-64-1, 40-42 cm. 2, 12. Sample 612-66-2, 40-42 cm, (2) Ceratiopsis diebelii (Alberti) Vozzhennikova, (12) Cannosphaeropsis utinensis O. Wetzel; emend. Duxbury. 3. Ceratiopsis sp. A, Sample 612-67-1, 40-42 cm. 4. Ceratiopsis sp. B, Sample 612-68-3, 44-46 cm. 5. Coronifera oceanica Cookson and Eisenack, Sample 612-67-3, 40-42 cm $\times 600$. 6. Areoligera senonensis Lejeune-Carpentier, Sample 612-67-5, 40-42 cm. 7. Bacchidinium sp.?, Sample 612-66-3, 40-42 cm, $\times 600$. 8. Cordosphaeridium fibrospinosum Davey and Williams, Sample 612-63-1, 40-42 cm. 9-10. Cribroperidinium sp. A, Sample 612-66-6, 40-42 cm. 11. Coronifera striolata (Deflandre) Stover and Evitt, Sample $612-71-2,40-42 \mathrm{~cm}, \times 600 . \quad 13$. Glaphrocysta ordinata (Williams and Downie) Stover and Evitt, Sample 612-61-1, 40-42 cm. 14. Cyclonephelium distinctum Deflandre and Cookson, Sample 612-69-5, 40-42 cm, $\times 600$. 


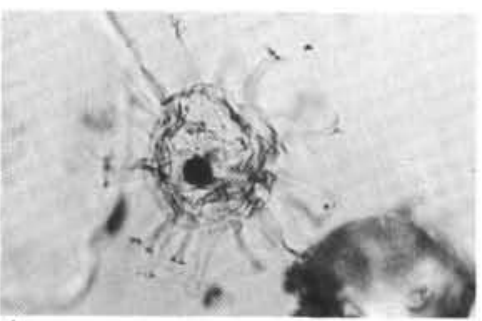

1

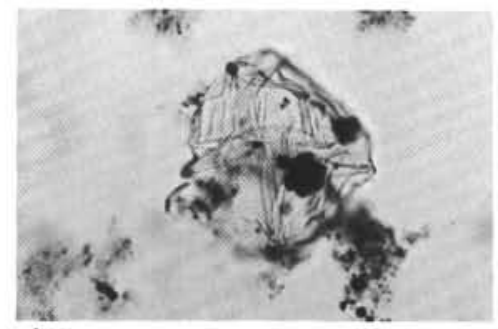

4

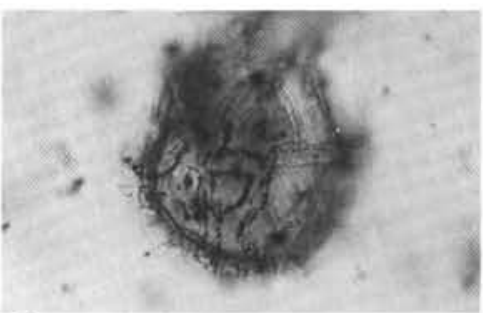

2

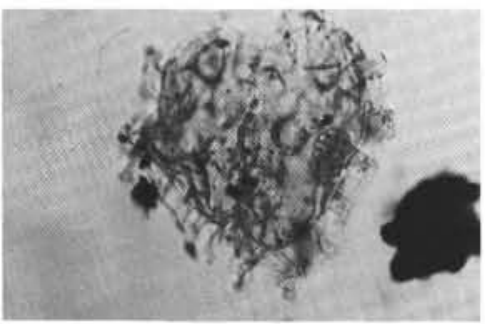

5

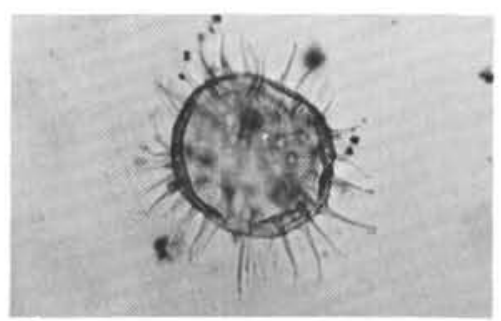

3

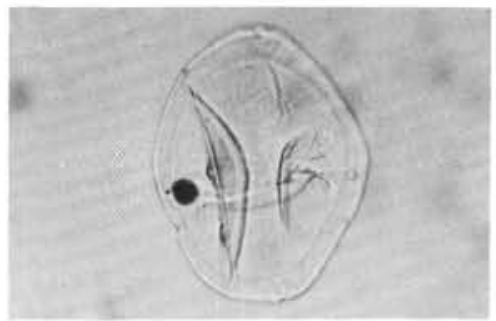

6

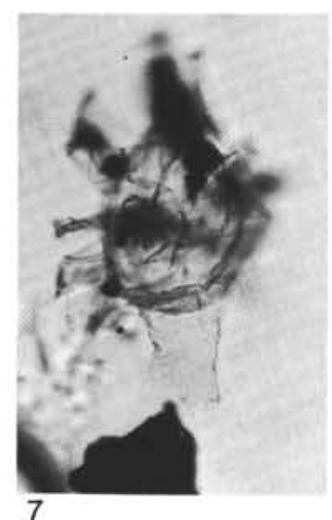

7

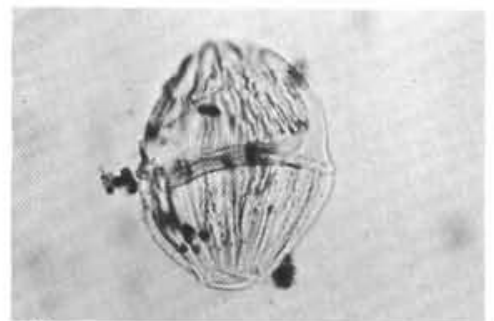

11

8
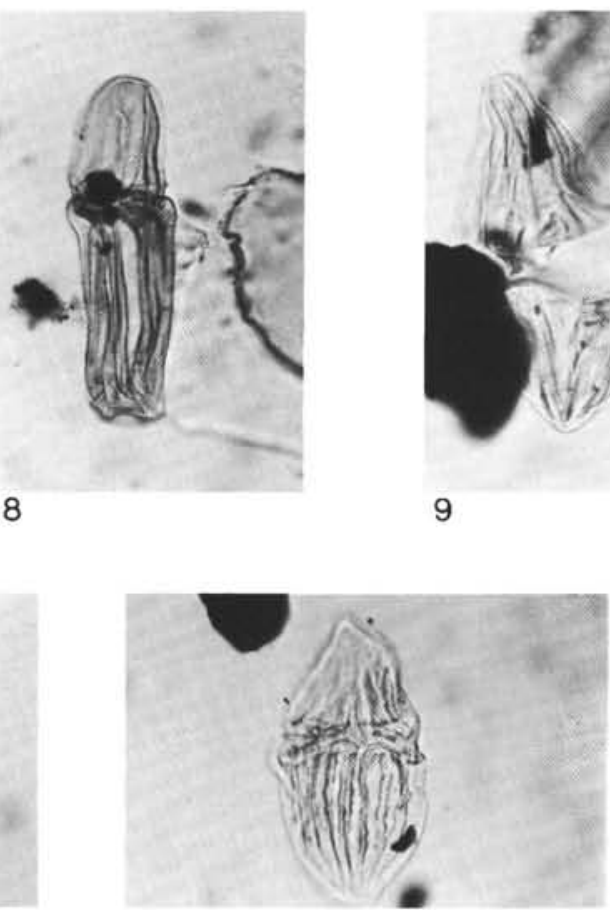

12

9
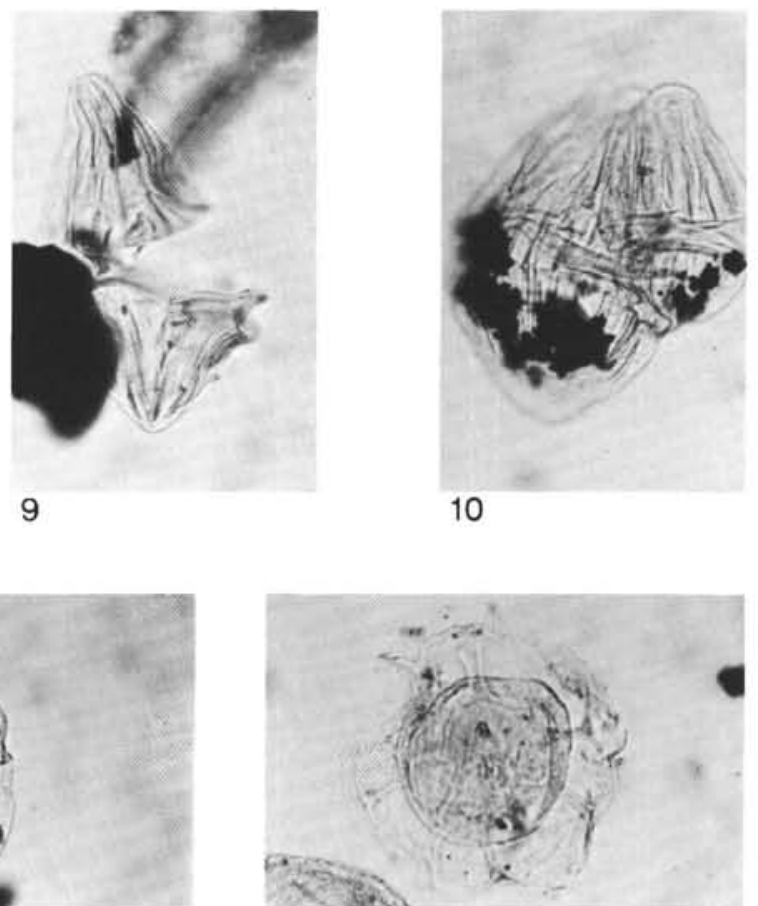

13

Plate 2. (Specimens photographed at $\times 600$ unless otherwise stated. All specimens photographed under normal transmitted light.) 1, 10. Sample 612-71-2, 40-42 cm, (1) Dapsilidinium? pumilum (Davey and Williams) Lentin and Williams, (10) Dinogymnium sp. B. 2. Leptodinium clathratum (Cookson and Eisenack) Sarjeant, Sample 612-71-6, 40-42 cm, $\times 400$. 3. Exochosphaeridium bifidum (Clarke and Verdier) Clarke et al., Sample 612-67-3, 40-42 cm, $\times 400$. 4, 8. Sample 612-69-2, 40-42 cm, (4) Dinogymnium cf. euclaense Cookson and Eisenack, (8) Dinogymnium digitus (Deflandre) Evitt et al. 5. Glaphrocysta retiintexta (Cookson) Stover and Evitt, Sample 612-72-6, 40-42 cm $\times 400$. 6. Eurydinium ingramii (Cookson and Eisenack) Stover and Evitt, Sample 612-67-6, 40-42 cm. 7. Hystrichokolpoma unispinum Williams and Downie, Sample 612-72-3, 40-42 cm, $\times 400$. 9. Dinogymnium microgranulosum Clarke and Verdier, Sample 612-72-5, 40-42 cm. 11. D. westralium (Cookson and Eisenack) Evitt et al., emend. May, Sample 612-61-2, 40-42 cm. 12. D. sp. A, Sample 612-67-2, 40-42 cm. 13. Membranilarnacia leptoderma Cookson and Eisenack) Eisenack, Sample 612-69-2, 43-45 cm. 


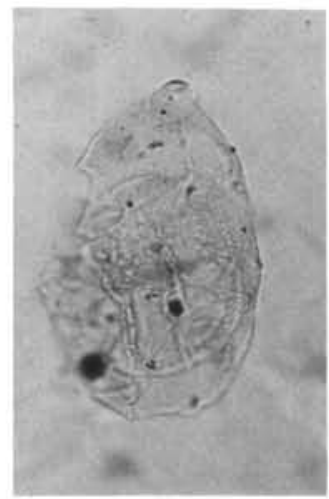

1

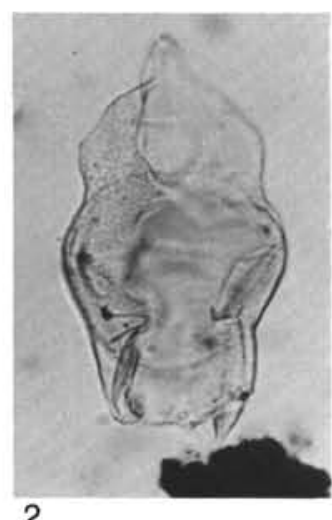

2

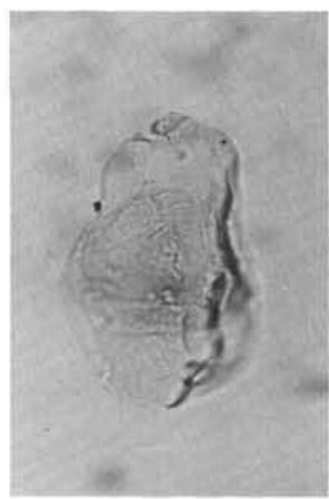

3

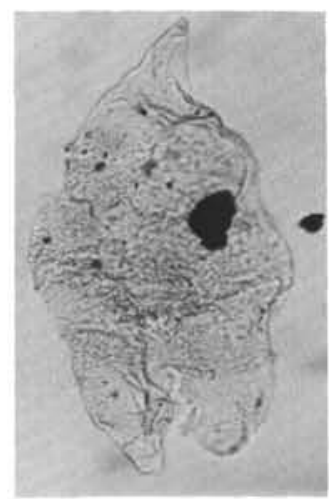

4

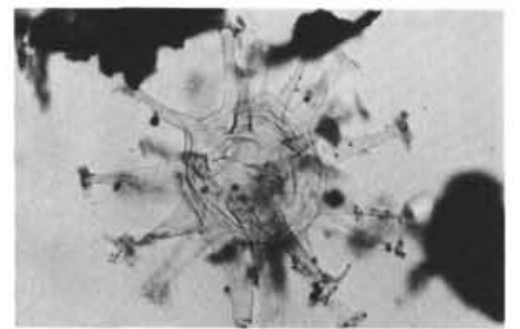

5

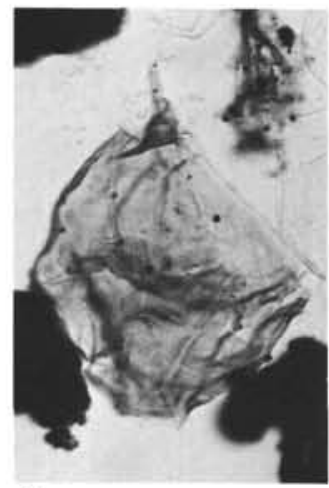

8

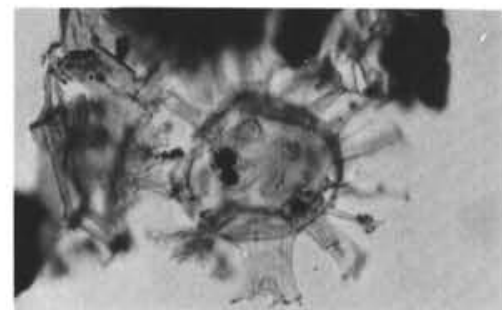

12

9

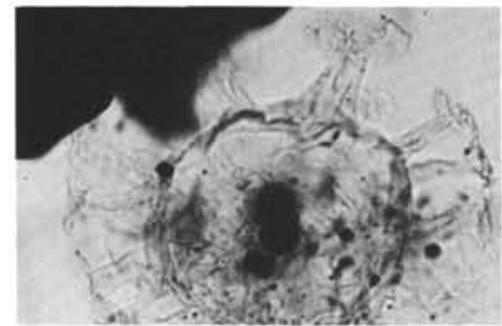

6
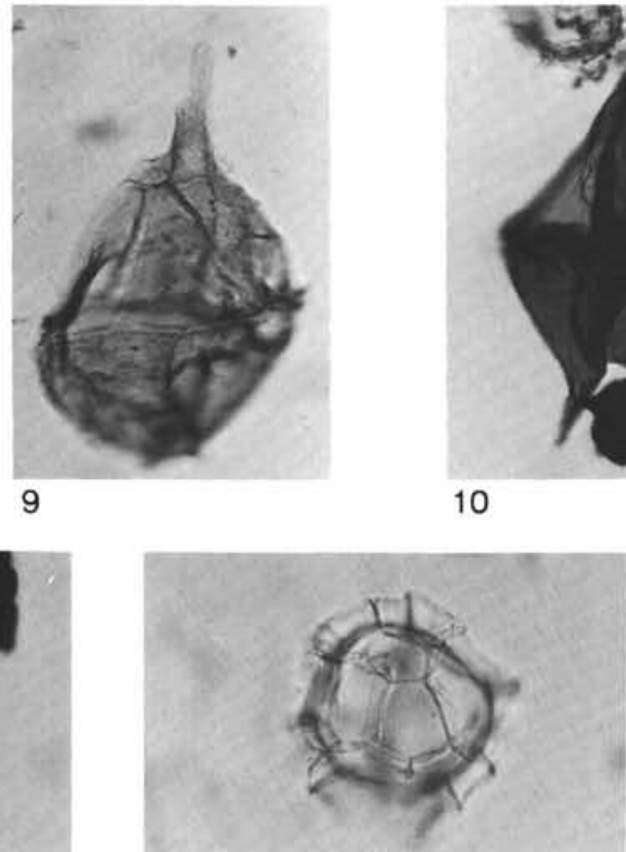

13

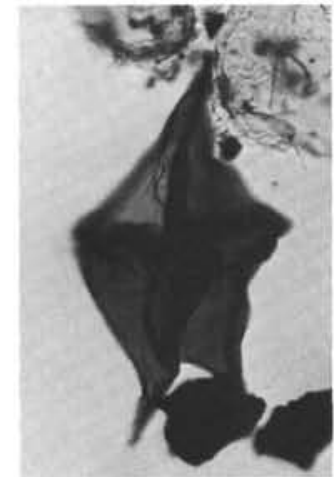

10

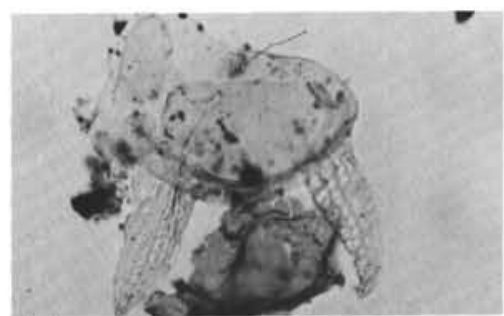

14

Plate 3. (Specimens photographed at $\times 400$ unless otherwise stated. All specimens photographed under normal transmitted light). 1. Isabelidinium belfastense (Cookson and Eisenack) Lentin and Williams, Sample 612-66-3, 40-42 cm, $\times 600$. 2. I. cooksoniae (Alberti) Lentin and Williams, Sample 612-69-2, 43-45 cm, $\times 600$. 3, 13. Sample 612-63-1, 40-42 cm, $\times 600$, (3) I. cretaceum? (Cookson) Lentin and Williams, (13) Impagidinium cristatum (May) Lentin and Williams. 4. I. thomasii (Cookson and Eisenack) Lentin and Williams, Sample 612-67-2, 40-42 cm, $\times 600$. 5. Hystrichosphaeridium tubiferum tubiferum (Ehrenberg) Deflandre; emend. Davey and Williams, Sample 612-72-6, 40-42 cm. 6. H. sp., Sample 612-70-3, 38-40 cm. 7. Hystrichostrogylon membraniphorum Agelopoulos, Sample 612-68-3, 44-46 cm, $\times 600$. 8. Gonyaulacysta? wetzelii (Lejeune-Carpentier) Sarjeant, Sample 612-72-3, 40-42 cm. 9. Cribroperidinium edwardsii (Cookson and Eisenack) Stover and Evitt, Sample 612-71-2, 40-42 cm. 10, 12. Sample 612-61-2, 40-42 cm, (10) Phelodinium tricuspis (O. Wetzel) Stover and Evitt, (12) Kleithriasphaeridium truncatum (Benson) Stover and Evitt. 11. Palaeocystodinium australinum (Cookson) Lentin and Williams, Sample 612-65-1, 40$42 \mathrm{~cm}$. 14. Odontochitina porifera Cookson, Sample 612-69-6, $45-47 \mathrm{~cm}$. 

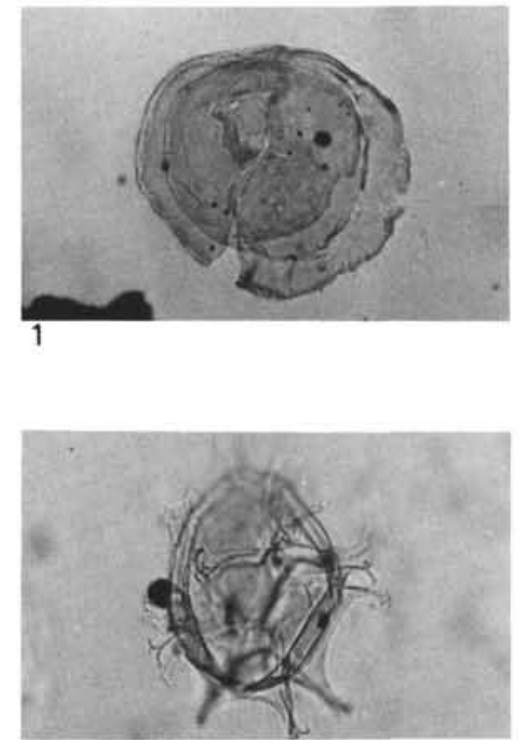

4

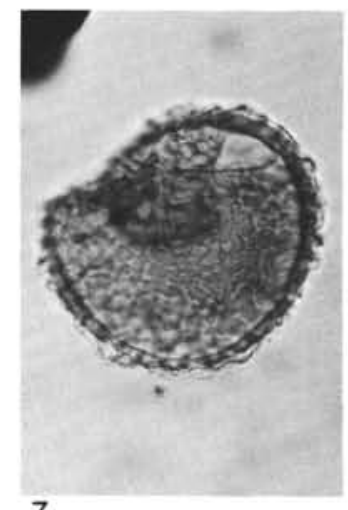

7

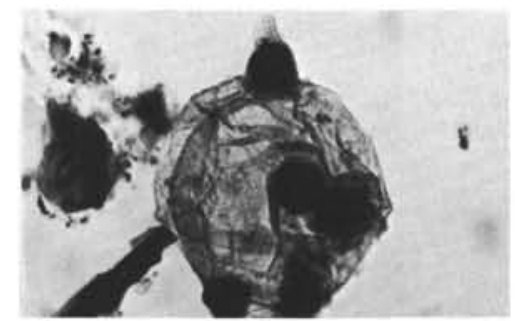

11

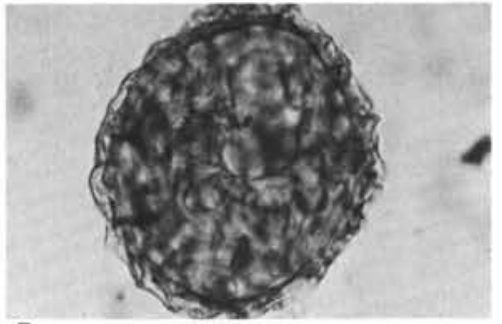

2

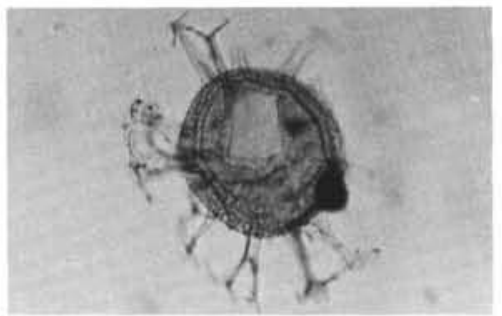

5

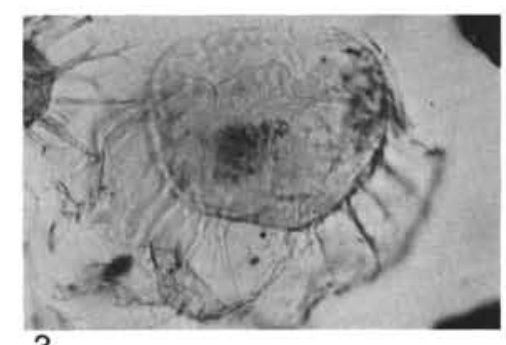

3

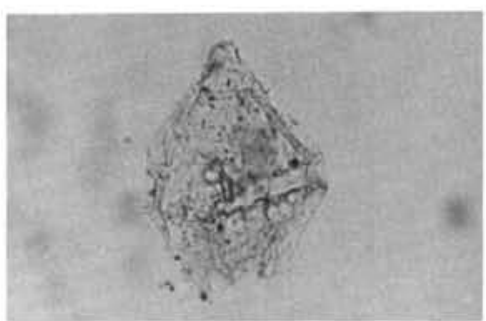

6

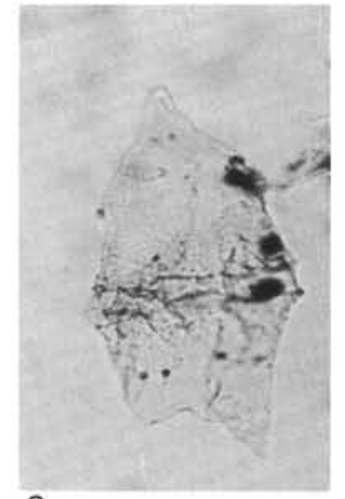

8

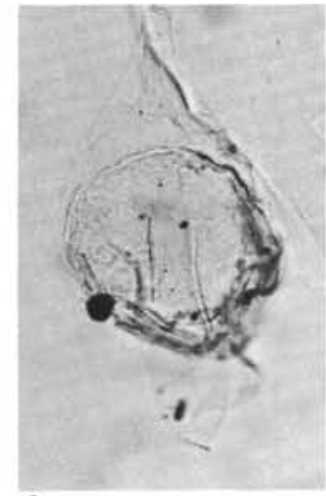

9

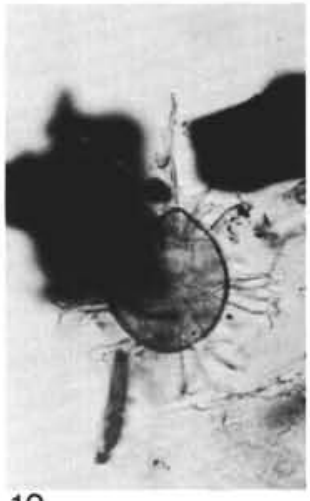

10

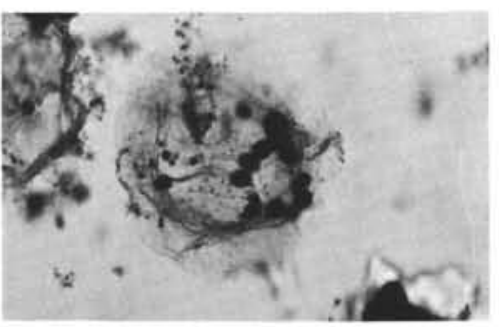

12

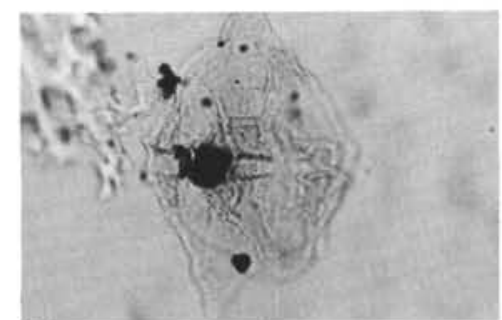

13

Plate 4. (Specimens photographed at $\times 600$ unless otherwise stated. All specimens photographed under normal transmitted light). 1. Paralecaniella indentata (Deflandre and Cookson) Cookson and Eisenack; emend. Elsik, Sample 612-71-2, 40-42 cm, $\times 400$. 2. Samlandia? angustivela (Deflandre and Cookson) Eisenack, Sample 612-69-2, 43-45 cm. 3. Cyclonephelium expansum Corradini, Sample 612-66-4, 40-42 cm, $\times 400$. 4, 7, 9. Sample 612-67-5, 40-42 cm, (4) Spiniferites ramosus (Ehrenberg) multibrevis (Davey and Williams) Lentin and Williams, (7) Pyxidinopsis sp.? (9) Trithyrodinium sp. 5. S. ramosus (Ehrenberg) reticulatus (Davey and Williams) Lentin and Williams, Sample 612-69-1, 40-42 cm, $\times 400$. 6. Spinidinium ornatum (May) Lentin and Williams, Sample $612-67-1,40-42 \mathrm{~cm}$. 8. Vozzhennikovia sp.?, Sample $612-71-5$, 40-42 $\mathrm{cm}$. 10. Spiniferites cornutus (Gerlach) Sarjeant, Sample $612-63-4,40-42 \mathrm{~cm}, \times 400$. 11. Senegalinium sp.?, Sample 612-72-1, 39-41 cm. 12. Dinopterygium sp.?, Sample $612-72-4,38-40 \mathrm{~cm}$. 13. Spinidinium sverdrupianum (Manum) Lentin and Williams, Sample 612-72-2, 42-44 cm. 